\title{
Philosophiques
}

\section{De quelques régimes de vérité (et de fausseté) littéraire}

\section{Jean-Marie Schaeffer}

Volume 40, numéro 1, printemps 2013

Littérature et connaissance

URI : https://id.erudit.org/iderudit/1018373ar

DOI : https://doi.org/10.7202/1018373ar

Aller au sommaire du numéro

Éditeur(s)

Société de philosophie du Québec

ISSN

0316-2923 (imprimé)

1492-1391 (numérique)

Découvrir la revue

Citer cet article

Schaeffer, J.-M. (2013). De quelques régimes de vérité (et de fausseté) littéraire. Philosophiques, 40(1), 9-21. https://doi.org/10.7202/1018373ar

\section{Résumé de l'article}

La question de la relation entre vérité et littérature se pose autrement selon qu'on aborde la littérature comme une forme d'art ou comme une forme de discours. Il faut aussi distinguer plusieurs régimes de vérité/fausseté, voire plusieurs types de réussite et d'échec littéraires qui ne peuvent peut-être pas tous être analysés en termes de vérité/non-vérité. À partir de là on peut envisager la relation entre valeur de vérité et fonction cognitive. d'utilisation que vous pouvez consulter en ligne.

https://apropos.erudit.org/fr/usagers/politique-dutilisation/ 


\title{
De quelques régimes de vérité (et de fausseté) littéraire
}

\author{
JEAN-MARIE SCHAEFFER \\ EHESS, Paris
}

\begin{abstract}
RÉSUMÉ. - La question de la relation entre vérité et littérature se pose autrement selon qu'on aborde la littérature comme une forme d'art ou comme une forme de discours. II faut aussi distinguer plusieurs régimes de vérité/fausseté, voire plusieurs types de réussite et d'échec littéraires qui ne peuvent peut-être pas tous être analysés en termes de vérité/non-vérité. À partir de là on peut envisager la relation entre valeur de vérité et fonction cognitive.
\end{abstract}

SUMMARY. - Answers to the problem of the relation of literature and truth differ according to whether one takes literature as a form of art or as a form of discourse. One must also distinguish various regimes of truth and falsity and various kinds of literary success or failures which cannot all be analysed in terms of truth and falsity. Once these points are examined on can deal with the relation between truth value and cognitive function.

La question des relations entre vérité et littérature m'est toujours apparue comme une entreprise particulièrement difficile, et ce pour plusieurs raisons. D'une part, les notions mises en relation sont elles-mêmes difficiles à identifier clairement, ou plutôt leur signification a tendance à varier fortement selon les auteurs. La vérité de l'être qui selon Martin Heidegger est révélée par le poème, "das Gedicht ", nous propose une figure de la vérité qui ne coïncide sans doute pas avec celle que l'on trouve à l'arrière-plan de la thèse frégéennerussellienne de la dénotation nulle de la littérature fictionnelle. La même variabilité s'observe du côté de la notion de «littérature »: le «Gedicht » heideggerien ne coïncide pas avec la «fiction» lue dans une perspective frégéenne ou russellienne.

Il y a par ailleurs souvent des affinités électives entre le type de théorie de la vérité que défend un philosophe et le genre littéraire qu'il pose comme incarnant la littérature ou l'art littéraire: la vérité-révélation a tendance à prendre le poème comme figure centrale de l'art littéraire, alors que les théories de la vérité-référence prennent plutôt comme modèle la fiction. De même les premières ont tendance à lire la littérature comme une synecdoque de l'art, alors que les secondes la traitent plutôt comme un type spécifique de discours. On verra que cette différence d'approche a des conséquences majeures quant à la façon même dont on pose la question de la vérité littéraire.

Enfin, de nos jours, la question de la relation entre littérature et vérité est souvent reformulée comme question de la fonction cognitive de la littérature. Or il n'est pas sûr que la valeur cognitive d'une œuvre littéraire, et plus généralement d'un dispositif artistique, dépende de sa valeur de vérité. Cela est dû au fait que, dans bien des œuvres littéraires, la fonction assertive 
du discours perd en importance par rapport à ses effets perlocutoires: dans ces cas, sa fonction cognitive ne se mesure pas tant par son adéquation par rapport à ce qui est représenté, donc par rapport à la vérité ou la fausseté de la représentation, que par sa capacité ou son absence de capacité à provoquer des actes cognitifs et des engagements émotifs chez celui qui le lit.

Il s'agit là d'un ensemble de questions très complexes que pour ma part je maîtrise très mal. Aussi me bornerai-je à proposer quelques distinctions qui auront rempli leur fonction si elles réussissent à montrer que la relation entre vérité et littérature n'est pas une mais multiple. Je me limiterai en fait à trois questions. J'essaierai d'abord de montrer en quel sens la question de la relation entre vérité et littérature se pose autrement selon qu'on y voit une instanciation de la question de la relation entre vérité et art, ou entre vérité et discours, donc selon qu'on aborde la littérature comme une forme d'art ou comme une forme, ou un ensemble de formes, de discours. En deuxième lieu j'aimerais consacrer quelques réflexions au caractère plurivoque de la notion de «littérature» et donc sans doute à la nécessité de distinguer plusieurs types de vérité/fausseté, ou plusieurs régimes de vérité/fausseté, voire plusieurs types de réussite et d'échec littéraires qui ne peuvent peut-être pas tous être analysés en termes de vérité/ non-vérité. Enfin, pour finir, je dirai quelques mots de la question des relations entre valeur de vérité et fonction cognitive. C'est une question à propos de laquelle je ne vois pas clair du tout, et ce que je proposerai sera à prendre avec précaution.

Il n'est sans doute pas inutile de justifier la présence de la notion de «fausseté » qui figure dans le titre de l'article. Il me semble que si nous admettons que la question de la vérité est pertinente pour la littérature, donc si nous admettons que la littérature a une potentialité véridictionnelle, alors nous devons admettre que la notion de non-vérité - que ce soit sous la forme de l'erreur ou du mensonge - est elle aussi un critère pertinent. Car on voit mal comment là où il y a un potentiel de vérité, il pourrait ne pas y avoir aussi un potentiel de fausseté. Or souvent les avocats les plus décidés de l'importance de la vérité littéraire procèdent autrement: ils excluent du champ de la littérature les œuvres dont ils contestent la portée du point de vue de la vérité. Dans ce cas, être une œuvre littéraire et donner accès à la vérité relève de la tautologie. Cette façon de procéder est précisément celle qu'on trouve chez ceux pour qui la vérité littéraire n'est qu'une synecdoque de la vérité de l'art, ce qui m'amène à la première des trois questions que je voulais aborder.

\section{La littérature comme art ou comme discours?}

Est-ce que la question des relations entre vérité et littérature est une instanciation de la question de la relation entre vérité et art, ou entre vérité et discours? Si cette question est importante, c'est parce que la thèse selon laquelle la question pertinente était celle des relations entre vérité et art a dominé pendant presque deux siècles les discussions philosophiques et littéraires. Or 
cette façon de voir a des conséquences importantes pour la notion même de "vérité » qu'on pose comme pertinente.

C'est un fait que dans la tradition philosophique continentale, depuis Kant en tout cas, la question des relations entre vérité et littérature est posée majoritairement non pas comme une question des relations entre la vérité et un type de discours, mais comme une question des relations entre la vérité et l'art. Autrement dit, c'est la littérature en tant que forme d'art qui est en jeu, et non pas la littérature en tant que type de discours. La littérature est bien entendu une forme d'art, et ce n'est pas cela qui pose problème. En revanche ce qui peut poser problème, c'est que dans cette façon de voir la question de sa vérité n'est pas posée dans une perspective comparative qui mettrait en relation différents types de discours, mais dans une perspective comparative qui met en relation différents types de vérité, dont la vérité artistique serait un type spécifique. La relation entre vérité et littérature apparaît alors comme une simple exemplification de la relation entre vérité et art.

Il faut noter d'abord qu'une telle théorie n'a de sens que si la notion d'art est une notion génériquement assez cohérente et unie pour qu'on puisse lui imputer un ensemble de propriétés communes du point de vue de la question de la vérité, propriétés qui seraient communes à toutes les formes d'art. Est-ce qu'on peut poser une telle cohérence générique? Je laisserai de côté la question fondamentale qui concerne la robustesse épistémique de la notion d' "art ", robustesse qui ne va pas de soi, dans la mesure où les classes qu'elle subsume au fil du temps sont très variables, ce qui peut faire douter de son caractère épistémique stable. Mais même si on accepte l'hypothèse que la notion générale d' " art » est une notion cognitivement fructueuse, il n'est pas sûr que l'unité susceptible de garantir ce caractère pertinent puisse se rapporter à une commune dimension véridictionnelle des différents arts.

D'abord, ce ne sont pas tous les arts qui possèdent une dimension propositionnelle: c'est le cas de la musique, même lorsqu'elle est à programme, de l'architecture, même lorsqu'elle est mimétique ou symbolique, et de la peinture, même lorsqu'elle est figurative. Or, dès que l'on sépare la notion de vérité du champ propositionnel, elle devient difficile à saisir et à circonscrire. On pourrait tenter de dépasser cette difficulté en notant que la question de la vérité ne se pose pas seulement à propos des représentations discursives, mais à propos de toutes les représentations: par exemple une représentation picturale pourrait être vraie au sens où elle représenterait correctement ce qu'elle prétend représenter. Mais sur quel plan cette question se pose-t-elle? Prenons le cas d'un tableau figuratif, par exemple une crucifixion. Sur quel plan la question de la vérité est-elle pertinente? Est-ce sur le plan de la mimésis perceptuelle générique? Sur le plan de la référence singulière? Sur celui de la signification symbolique? Bref, la question de la vérité d'une représentation visuelle figurative se pose-t-elle sous la forme du réalisme ou du naturalisme mimétique, relativement à son degré de fidélité à cet événement singulier et supposé réel que fut la crucifixion du Christ ou au niveau symbolique de 
la crucifixion, donc à un niveau où les deux premiers régimes de vérité ne sont pas pertinents. On admettra pour le moins que, selon le niveau qu'on privilégiera, la notion de vérité risque d'avoir des conditions de satisfaction fort différentes.

En tout état de cause, il n'est pas sûr que tous les arts soient représentationnels au sens technique du terme, c'est-à-dire se rapportent à une réalité extérieure: on connaît les débats sans fin à propos du statut représentationnel ou non de la musique, mais la même chose peut être dite de l'architecture et bien entendu des arts décoratifs. En fait, si l'on excepte les architectures mimétiques et la musique à programme, qui sont des cas dans lesquels le contenu représentationnel est donné de l'extérieur (par le titre dans le cas de la musique ou par ce qui est imité dans le cas de l'architecture), l'idée selon laquelle les œuvres de ces arts auraient un contenu représentationnel déterminé se heurte, comme Hegel notamment l'avait bien vu, au fait que ces arts ne disposent pas de véhicule sémiotique susceptible de donner lieu à des contenus déterminés du point de vue sémantique. La réussite d'une pièce de musique ou d'une œuvre architecturale semble se mesurer plutôt en termes de fluence ou de difficulté perceptive, de simplicité ou de complexité formelle, de justesse et de richesse expressive ou émotive, etc., qu'en termes d'adéquation véridictionnelle.

Mais le véritable problème est ailleurs. Si on pose la question de la vérité littéraire en y voyant une instanciation de la vérité en/de l'art, alors la manière dont l'art est pensé eu égard à la question de la vérité va du même coup déterminer ce qu'on dira à propos de la littérature. Or la manière selon laquelle la question de la vérité de l'art est posée depuis deux siècles est largement déterminée par une thèse très forte concernant la vérité artistique, thèse qui a été élaborée par la tradition philosophique allemande qui va du romantisme à Heidegger, mais qui a essaimé loin au-delà de la philosophie pour devenir un lieu commun de la critique artistique. Autrement dit, l'idée selon laquelle les arts dans leur diversité entretiendraient foncièrement la même relation avec la problématique de la vérité n'est le plus souvent que l'autre face de la thèse selon laquelle les arts relèveraient d'un type de vérité spécial, c'est-à-dire qu'il y aurait un rapport au vrai qui non seulement serait commun aux arts mais leur serait aussi radicalement propre et qui se distinguerait de la vérité de type propositionnel ou représentationnel au sens technique (par exemple de fidélité à la perception). La thèse selon laquelle il y aurait un type de vérité spécifique à l'art est souvent avancée en faisant référence aux désaccords entre Platon et Aristote concernant la fonction cognitive de la mimèsis. Mais en réalité, pour Aristote en tout cas, la mimésis ne coïncide pas avec la poièsis, au sens où il existe des arts non mimétiques. Certes, il n'y a pas de doute que, dans la perspective platonicienne, la question de la vérité de la mimèsis est posée dans le cadre d'une interrogation plus générale sur la poièsis artistique dans ses relations avec la thèoria du philosophe, donc avec la figure canonique de l'incarnation du vrai. Il est vrai 
qu'Aristote de son côté la pose explicitement dans le cadre d'une interrogation sur la distinction entre la vérité de la mimèsis et la vérité historique. En ce sens il admet bien une vérité sinon spécifiquement artistique du moins spécifiquement mimétique. Mais nous sommes ici dans le domaine de la mimésis qui n'est qu'une province de l'art pour les deux philosophes antiques.

Cela ne signifie pas que la thèse d'une vérité qui serait spécifique à l'art n'ait pas de racines grecques. En fait il est probable qu'elle trouve son origine dans la théorie platonicienne du beau selon laquelle les objets beaux sont beaux dans la mesure où ils participent à l'Idée du Beau. Or, chez Platon l'Idée du Beau est conjointe à celle du Bien mais aussi à celle du Vrai. Cela nous donne à la fois un critère auquel un objet doit satisfaire pour pouvoir être qualifié de beau (il doit se rapporter au Beau transcendant) et un lien entre le Beau et le Vrai (ainsi que le Bien), au sens où le Beau en tant qu'Idée est une figure du Vrai. Cependant, pendant longtemps la théorie du Beau a été en premier lieu non pas une théorie de l'art mais une théorie de la belle apparence, par exemple de la beauté corporelle. Le lien entre théorie du beau et théorie de l'art ne s'est établi que peu à peu en plusieurs étapes, dont le plotinisme de la fin de l'antiquité et le néoplatonisme renaissant sont les moments les plus importants avant le romantisme. C'est en effet avec ce dernier, qui inscrit la théorie de l'art dans une ontologie platonicienne de l'être et de l'apparence, que le Beau comme figure du Vrai et l'Art vont être identifiés l'un à l'autre, donnant naissance à la thèse d'une vérité spécifiquement artistique réputée être de nature extatique, c'est-à-dire capable grâce à un type d'intuition intellectuelle de nous mettre en relation directement avec l'être. En cela la force cognitive de l'art dépasse les vérités propositionnelles à référence intramondaine, par exemple les vérités scientifiques: celles-ci ne nous mettent en relation qu'avec le monde de l'apparence. Cette idée d'une vérité artistique de nature extatique (abolissant notamment la distinction entre le sujet connaissant et l'objet) a elle-même connu de nombreuses péripéties, en particulier quant à la question de la hiérarchie entre la vérité artistique et la vérité philosophique qui elle aussi prétendait atteindre directement l'être en se dissociant radicalement des savoirs empiriques portant sur le monde phénoménal. Mais à travers toutes ces vicissitudes, la structure conceptuelle de base est restée la même, que ce soit chez Schelling, Hegel ou Schopenhauer, ou chez Heidegger. Chez ce dernier par exemple il n'y a pas de doute que la cohérence de la notion de «Kunstwerk» eu égard à la question d'un rapport proprement artistique à la vérité est fondée sur l'hypothèse d'une figure de la vérité qui serait propre à l'art et de même dignité que le vrai philosophique.

On comprend pourquoi la théorie de la vérité artistique comme vérité extatique est foncièrement antimimétique. En effet pour poser un type de vérité extatique qui serait propre à l'art, il faut dissocier cet art, y compris l'art littéraire, du type de vérité susceptible de le rapprocher le plus du domaine de la vérité propositionnelle, ce qui est évidemment le cas de la mimèsis. La 
théorie romantique, reprenant la notion renaissante de fantasia et réinterprétant la notion kantienne d'imagination productive voit dans le vrai artistique non pas le reflet d'un modèle intramondain, mais la libre construction d'un univers idéal conçu comme expression directe de l'être. On ne se situe donc plus dans une conception de la vérité comme vérité-correspondance (ce qui était le cas de la théorie mimétique) mais bien dans une conception de la vérité comme révélation et participation: ce qui compte n'est pas la correspondance entre la représentation et une réalité supposée pré-existante qu'elle imite, mais la fonction performative de l'œuvre eu égard à la vérité ontologique. L'idée heideggerienne selon laquelle les poètes sont ceux qui ouvrent le temps historique de leur peuple, qui instaurent la vérité historique de ce peuple, n'a de sens que parce que Heidegger situe l'œuvre poétique dans une conception de la vérité artistique comme vérité performative.

Mon but ici n'est pas de prendre parti pour ou contre cette conception sans conteste exaltante de la vérité artistique. Elle m'intéresse uniquement en ce qu'elle permet de comprendre que si nous posons la question des relations entre vérité et littérature dans son cadre, donc dans le cadre où la littérature vaut comme instanciation de l'art et non pas comme instanciation d'une modalité de discours, alors nous ne saurions nous la poser correctement qu'à condition de disposer d'une théorie convaincante de la vérité artistique conçue: a) comme type de vérité commune à tous les arts et donc indifférente aux spécificités représentationnelles des différents types d'art; et b) comme type de vérité spécifique aux arts, donc susceptible d'être distinguée de la vérité propositionnelle. Comme j'ai essayé de le montrer, ces deux conditions restent valables même si on n'adhère pas à l'idée selon laquelle cette vérité spécifique serait de l'ordre d'une intuition extatique. Ces conditions me semblent difficilement pouvoir être satisfaites en raison du caractère épistémiquement peu robuste de la notion d'art. Cette absence de robustesse est liée au fait que la cristallisation historique de la classe extensionnelle que notre culture a fait correspondre à cette notion est due non pas à la découverte d'un ensemble de propriétés internes communes à ces objets, mais à leur inscription commune dans un même type d'usage, ayant donc trait à une propriété relationnelle, en l'occurrence celle de la relation esthétique. Si tel est le cas, alors la notion d' " art » est unifiée primordialement non pas par une propriété qui lierait les œuvres à ce qui est, mais plutôt par une propriété qui les lie à leurs usages.

\section{Littérature et fiction}

J'en viens maintenant à ma deuxième question, qui est celle de savoir si la notion de "littérature " est susceptible de nous donner un champ d'objets stable par rapport auquel la question de la vérité pourrait recevoir une réponse déterminée. La perspective ici n'est plus celle de la littérature comme instanciation de l'art mais comme type de discours, donc comme quelque chose qui a, ou du moins est susceptible d'avoir une structure illocutoire assertive. 
Ici, il ne s'agit plus d'affirmer une vérité extatique métapropositionnelle pour la littérature, mais de s'interroger sur ses rapports avec les autres discours eu égard à la question de la vérité comme propriété, valeur propositionnelle. Voilà sans doute une question plus facile à manier: dès lors que ce qui est en jeu est un type, ou des types de discours, nous nous trouvons en terrain familier, puisque quoi qu'elle soit par ailleurs, la vérité est un attribut que nous accordons prioritairement à des propositions.

En fait, si la question nous paraît simple, c'est en partie grâce à une confusion concernant l'objet sur lequel elle porte. La notion de «littérature», je l'ai déjà dit, est loin d'être univoque. Elle est plutôt à géométrie variable. Cela se traduit notamment par le fait que lorsque nous prétendons nous interroger sur la relation entre vérité et littérature, nous nous interrogeons en réalité sur la question des relations entre vérité et fiction. On en a un exemple particulièrement révélateur dans le livre classique de Peter Lamarque et Stein Haugam Olsen Truth, Fiction and Literature (Oxford Clarendon Press, 1994), dans lequel ils défendent leur thèse bien connue selon laquelle les notions de vérité, de connaissance, etc., ne sont pas pertinentes pour décrire les œuvres littéraires. Comme le titre l'indique, ils affirment qu'ils distinguent explicitement entre fiction et littérature, notamment en traitant la première comme une catégorie descriptive (intentionnaliste pour être tout à fait précis), et la seconde comme une catégorie évaluative. Mais en réalité ils définissent la littérature par la notion de "mimèsis", et même si on peut discuter sans fin quant à savoir si cette notion recouvre exactement la notion de «fiction", il n'y a guère de doute que les deux sont très proches l'une de l'autre. D'ailleurs les deux auteurs se servent pour l'essentiel de récits de fiction pour développer leur théorie de la littérature.

On comprend pourquoi nous avons tendance à nous rabattre sur la fiction et pourquoi nous tendons à y voir la synecdoque de la littérature comme telle. Du fait de la mimésis formelle qui, à des degrés divers, relie la fiction aux récits factuels et par extension aux propositions descriptives, la fiction nous met face à une situation de parallélisme syntaxique avec le domaine discursif par excellence du vrai et du faux dénotationnel dans la vie vécue. Si la question de la vérité littéraire se réduit à celle de la vérité de la fiction, il semblerait qu'on puisse lui donner une réponse précise. Et effectivement, du point de vue d'une acception technique de la notion de vérité littéraire, les travaux les plus fructueux et les plus importants concernent bien le champ de la fiction, qu'il s'agisse de la théorie aristotélicienne de la mimèsis ou des descriptions actuelles en termes de contrefactuels ou de mondes possibles. Comme le montrent les thèses de Lamarque et Olsen, la thèse selon laquelle la fiction aurait une dimension véridictionnelle est loin d'être acceptée par tout le monde, mais tous ceux qui reconnaissent une portée cognitive à la fiction voient en général dans les théories des contrefactuels et des mondes possibles des alliés inestimables. 
Cependant, même si la délimitation de la notion de littérature est difficile à tracer de manière précise, il serait certainement extravagant de notre part de vouloir l'identifier à celle de la notion de fiction ou de mimèsis. Le champ de cette dernière est à la fois plus large et plus restreint que celui de la littérature. Il est plus large parce que la fiction n'est pas limitée à la littérature, puisqu'on en trouve aussi dans d'autres arts, dont la peinture et le cinéma. Ce qui pose d'ailleurs la question de savoir si la fiction, y compris littéraire, peut être réduite à un fait propositionnel. Certaines approches du point de vue de la simulation mentale auraient plutôt tendance à rapprocher son mode de fonctionnement de processus d'immersion imaginative synesthésique sous-déterminée par le contenu propositionnel de l'œuvre. Mais le champ de la fiction est aussi plus restreint que celui de la littérature, parce que toutes les œuvres littéraires ne sont pas fictionnelles ou mimétiques: il suffit ici de penser à la prose oratoire, aux récits de voyage, aux autobiographies, aux mémoires, etc.

C'est ce dernier point qui m'intéresse ici: vouloir limiter le champ des œuvres littéraires à celui des œuvres de fiction constitue un coup de force. Peut-on en conclure sans autre forme de procès que lorsqu'une œuvre littéraire n'est pas fictionnelle elle relève automatiquement du tribunal de la vérité et de la fausseté, autrement dit qu'elle est une variante littéraire de l'assertion sérieuse? Ce qui signifierait qu'eu égard à la vérité la littérature relèverait soit de la logique des mondes possibles, soit de la véridiction standard qui caractérise les descriptions et assertions non littéraires. Plusieurs raisons plaidant contre une telle dichotomie du littéraire qui permettrait une répartition entre textes de fiction et textes factuels. Je me limiterai ici à en énumérer deux.

La première est que beaucoup de textes que nous traitons comme des textes littéraires canoniques sont des textes qui ont des prétentions référentielles et plus largement sérieuses, mais pour lesquels malgré cela nous ne posons pas (ou plus) la question de leur pertinence du point de vue de la vérité référentielle. Autrement dit, la suspension de la question de la validité référentielle n'est pas réductible à la forme qu'elle prend dans la fiction. Il suffit de penser à l'art oratoire. Prenons les sermons de Bossuet: pour leur auteur ces textes possédaient une dimension véridictionnelle centrale, seule susceptible de garantir leur puissance exhortative. Tout en sachant cela, nous les lisons presque tous aujourd'hui en neutralisant cette double prétention. Pourtant nous ne les lisons pas comme des fictions: nous ne pensons pas par exemple que la dimension exhortatoire est seulement simulée ou que les assertions générales sont seulement des assertions feintes. Nous savons et nous acceptons qu'il s'agit d'un discours qui prétend à la véridiction et se veut exhortatif, simplement nous ignorons en quelque sorte ces deux prétentions. Cela ne signifie pas non plus nécessairement que nous lisons les sermons uniquement sous l'angle formel, par exemple sous l'angle stylistique. Nous pouvons fort bien apprécier par exemple leur force persuasive, leur 
subtilité d'argumentation, voire la justesse de l'image de la vie humaine qu'ils tracent. Mais cette justesse, nous pouvons l'apprécier sans adhérer à aucune des propositions théologiques dont pourtant elle se nourrit et que le texte asserte explicitement. Autrement dit, nous pouvons dissocier cette justesse de la vérité ou de la fausseté des assertions théologiques ou autres. Et pourtant, cette justesse relève pour nous du régime du sérieux et non pas du régime de la feintise ludique, du faire-semblant. On peut penser que des notions telles que la justesse ou la profondeur d'une vision ont affaire avec la vérité, mais en tout état de cause elles ne se laissent pas traduire en termes de vérités contrefactuelles ou de mondes possibles. Une vision que nous estimons juste ou profonde est une vision qui trouve des points d'application dans notre réalité vécue, et en ce sens on peut supposer qu'elle possède un caractère de véracité, mais la mise en relation de ce caractère avec la notion de vérité au sens référentiel du terme semble difficile.

La deuxième raison qui plaide contre une identification de la littérature à la fiction est qu'il existe de nombreuses œuvres littéraires, et en particulier de nombreux textes poétiques, pour lesquels le niveau du traitement dénotationnel comme tel, que cette dénotation soit fictionnelle ou non, n'occupe plus la place décisive qui est la sienne dans le discours non littéraire. Cette question de la hiérarchie des niveaux constitutifs de l'œuvre littéraire qui a été étudiée notamment par Roman Ingarden ne devient visible que lorsqu'on déplace le regard des œuvres conçues comme objets syntaxiques (à la Goodman) vers la question du traitement mental des œuvres. Si on déplace ainsi le regard, on constate qu'en poésie lyrique la hiérarchie des fonctions s'inverse souvent par rapport aux hiérarchies généralement dominantes hors du champ littéraire: la figure et le fond s'inversent en quelque sorte. Ainsi, dans beaucoup de poèmes, la fonction illocutoire comme telle n'est plus «foregrounded» : c'est plutôt la fonction perlocutoire sous la forme de l'effet émotif produit chez l'auditeur ou le lecteur qui est la fonction dominante. La couche représentationnelle - descriptive si l'on veut - est certes fonctionnelle, mais elle l'est non pas tant dans sa fonction assertive ou feinte qu'en tant qu'inducteur de représentations émotivement saturées.

Ces modes de fonctionnement, qu'il s'agisse de celui de l'art oratoire ou de celui d'une partie importante de la poésie lyrique sont irréductibles à la fois à la fiction et au discours factuel parce que la question de la dénotation, voire de la représentation, donc la relation avec une réalité extérieure y est moins importante que les effets perlocutoires. La représentation ou la description, la forme assertive propositionnelle n'y ont pas pour fonction de représenter un univers, feint ou réel, qui serait à apprécier comme tel dans sa valeur de vérité; elles engagent le lecteur à s'immerger dans une dynamique immersive qui, dans certains cas - qu'on pense par exemple à la poésie de Rimbaud -, ne saurait plus être évaluée en tant qu'univers, fût-il contractuel, mais primordialement en tant que dynamique d'associations. 
Est-ce que la question de la vérité se pose réellement dans un tel cas? Le poème ne s'accomplit-il pas dans l'expérience mentale qu'il provoque?

\section{Vérité et fiction}

Pour clore j'aimerais ajouter quelques mots concernant la dernière question: celle des relations entre la question de la vérité et celle de la fonction cognitive des œuvres littéraires. Est-ce que pour avoir une fonction cognitive, donc pour donner lieu à un processus d'apprentissage qui agrandit mes ressources cognitives, une œuvre littéraire doit être "vraie» ?

Je prendrai d'abord un cas emprunté à un domaine fort éloigné de la littérature, celui des jeux vidéos. On peut considérer que les jeux vidéos sont une "forme d'art ", parce que du point de vue poético-générique ils relèvent manifestement de la tecknè artistique et plus précisément du vaste champ des arts de la fiction (audiovisuelle). En même temps, du moins à première vue, en ce qui concerne leur valeur de vérité, les jeux vidéos semblent faire partie des types d'activités qu'on peut négliger. D'autant plus que les jeux dont il s'agira dans mon exemple sont des jeux de combat, qu'on considère généralement comme étant non seulement les plus frustes mais encore les plus débilitants dans toute la panoplie des jeux. Cette impression est difficilement contestable tant qu'on s'intéresse au niveau des univers fictifs mis en scène et des actions que le joueur peut entreprendre pour changer ce monde, action qui se limite en gros à tuer le plus d'adversaires possibles. Mais il en va autrement dès qu'on se tourne vers la question des modalités de la représentation, ou, pour être plus précis, vers celle des modalités d'interaction visuelle entre le joueur et l'espace du jeu.

Ce point a été démontré dans une étude menée par A. S. Shawn Green et Daphné Bavelier ${ }^{1}$. Les auteurs se posaient la question de savoir si la pratique massivement répandue des jeux vidéos a des conséquences sur les compétences motrices et perceptives des individus qui s'adonnent à cette pratique. En effet, en règle générale le fait d'exposer de façon récurrente un organisme à un environnement visuel changé résulte dans une réorganisation plus ou moins grande de son système visuel, réorganisation qu'on désigne par l'expression d' «apprentissage perceptuel». Pourquoi n'en irait-il pas de même en ce qui concerne les jeux vidéos? Et effectivement, Green et Bavelier ont montré que la pratique des jeux vidéos est bien capable de transformer toute une série de compétences visuelles généralistes. Le caractère innovant - et élégant — de l'étude réside dans le fait qu'au lieu de développer un dispositif expérimental spécifique avec des séances d'entraînement à une tâche artificielle dont on mesure ensuite les effets, les auteurs ont comparé directement les compétences de deux populations, une de joueurs de jeux vidéos et une de non-joueurs, en les soumettant aux tests de compé-

1. A. S. Shawn Green et Daphné Bavelier, «Action Video Game Modifies Visual Selective Attention", Nature, vol. 423, 29 mai 2003, p. 534-537. 
tence traditionnels sans les soumettre à un entraînement préalable. De ce fait, l'étude a pu mesurer directement l'effet de la pratique des jeux vidéos sur des compétences acquises à travers une pratique ludique dont le but n'était pas l'acquisition de ces compétences. Autrement dit, les compétences nouvelles ont été acquises sans visée intentionnelle dirigée vers leur acquisition. L'étude a montré de façon tout à fait convaincante que du point de vue des ressources visuelles attentionnellement disponibles, de leur activation, de leur distribution spatiale à travers le champ visuel mais aussi du point de vue des capacités d'intégration temporelle, une pratique régulière de jeux vidéos d'action aboutit à un véritable apprentissage perceptuel. Par ailleurs, les deux auteurs ont pu montrer que l'apprentissage était généralisable au-delà de la tâche pratiquée (le jeu vidéo) et restait opératoire même après plusieurs mois de cessation de toute pratique. On peut en conclure que la pratique de l'attention visuelle est, dans certaines conditions du moins, capable de déclencher un processus d'auto-apprentissage aboutissant à des compétences généralisables, c'est-à-dire ne restant pas limité à la tâche qui l'a déclenché.

L'objection qui se présente immédiatement est que cette valeur cognitive relative à l'affinement de la discrimination visuelle n'est en rien liée au fait que le stimulus se trouve être un jeu vidéo. Autrement dit, même si l'on me concédait qu'un jeu vidéo peut être considéré comme une œuvre d'art, l'effet mis en évidence est indépendant du fait que le stimulus est de nature artistique. Un dispositif expérimental classique bien conçu aurait pu produire le même résultat. Cette objection s'accorde en réalité fort bien avec l'hypothèse qui est la mienne, à savoir que si — et dans la mesure où — une œuvre d'art possède une valeur cognitive eu égard à un processus cognitif mobilisé aussi par d'autres activités attentionnelles, le type de connaissance ou de compétence qu'elle apporte est du même type que celui apporté par les autres activités attentionnelles qui sollicitent ce même processus. L'objection rencontre donc l'idée selon laquelle il n'existe pas de processus cognitif qui serait spécifique au traitement mental des œuvres d'art et que donc il n'existe pas de cognition spécifiquement artistique. Mais en contrepartie, l'étude que je viens de résumer suggère aussi qu'une œuvre d'art peut produire des effets cognitifs à des niveaux très profonds, sans que la question de sa vérité représentationnelle soit pertinente.

Pourrait-on concevoir que des processus du même type sont en œuvre dans la littérature? Je prendrai le cas de la fiction, puisque c'est le domaine pour lequel la dimension de la vérité est sans conteste tout à fait pertinente. Le rôle cognitif de la fiction peut-il pour autant être identifié à ce type de fonctionnement?

Certes, les fictions transmettent, comme en passant, d'innombrables connaissances factuelles, bref, l'univers de fiction est rempli d'innombrables assertions qui sont vraies au sens le plus basique du terme. La raison en est simple: le mobilier de l'univers des représentations est commun pour une part non négligeable au monde réel et à l'univers de fiction. Ainsi, les romans 
nous transmettent toujours des informations factuelles que nous pourrions par ailleurs trouver dans des récits factuels: par exemple, innombrables sont les romans qui permettent au provincial ou à l'étranger d'acquérir des informations fiables sur Paris, sur tel ou tel de ses quartiers, sur la situation des gares, etc. En fait, la vraie difficulté n'est pas tant de transmettre des connaissances factuelles de ce type, elle est plutôt de réussir à ne pas en transmettre trop, comme en témoignent les romans fantastiques dont la difficulté principale réside dans la nécessité de bloquer la transmission d'informations factuelles référant au monde réel dans lequel nous vivons.

Cela dit, ce n'est certainement pas dans ce type d'information factuelle que réside la valeur de vérité de la fiction en tant que fiction: ces informations-là sont plutôt la trace de l'univers non fictionnel dans l'univers fictionnel, une trace qu'aucune fiction ne saurait expulser au-delà d'un certain degré sans risquer de devenir incompréhensible. En fait, pour s'interroger sur la valeur de vérité propre que peut revêtir une fiction, il faut se tourner vers l'univers fictionnel comme le font les théories des contrefactuels ou les théories des mondes possibles. Mais est-ce que le potentiel cognitif de la fiction littéraire peut être décrit de manière satisfaisante en se bornant à l'analyser du point de vue du statut logique des propositions fictionnelles? Essayons de nous détourner pour un moment de l'univers créé pour nous tourner vers les conditions qui régissent la dynamique modélisante qui donne naissance à la fiction. Pour qu'une représentation factuelle puisse être source de connaissance, il faut qu'elle soit vraie en termes de référence intramondaine, il faut que tout ce qu'elle affirme concernant la réalité existe effectivement tel qu'elle le dit. Il n'en va pas de même pour une fiction, puisque par définition elle ne prétend pas porter sur des faits réels. La seule contrainte qui s'exerce sur la modélisation fictionnelle est que le modèle fictionnel doit pouvoir être engendré conformément aux lignes de force qui répondent aux conditions de représentabilité exigée de toute expérience pour que nous puissions la vivre mentalement comme expérience. Il s'agit donc d'une contrainte purement formelle et non sémantique. Le récit de fiction n'est pas tant une image du monde qu'une exemplification virtuelle d'un être-dans-le-monde possible. En effet, l'univers fictionnel est mentalement vécu en situation d'expérience immersive dans un univers plutôt qu'en situation d'attitude judicatrice. Du même coup sa fonction cognitive n'exploite pas tant l'éventuel statut véridictionnel des croyances propositionnelles ou déclaratives qu'elle véhicule que son statut de quasi-expérience vécue: et comme c'est le cas de toutes les expériences vécues, son aspect cognitif relève de l'affinement d'un savoirfaire, d'un kow-how, plutôt que de l'acquisition de nouvelles connaissances déclaratives. La lecture de $\mathrm{La}$ Recherche augmente certes à bien des égards notre connaissance déclarative concernant la France du début du XX $\mathrm{XX}^{\mathrm{e}}$ siècle, mais sa vertu cognitive la plus puissante est de nature psychologique: menés par le narrateur et les personnages à travers les nuances les plus infimes des réactions émotives et du sentiment de soi nous acquérons à leur contact un 
surcroît de sensibilité et de pouvoir discriminant dans le champ des sentiments et de la connaissance de notre propre moi. Il faut d'ailleurs noter que ce gain cognitif est non détachable de l'expérience de l'univers fictif: contrairement au contenu déclaratif, il ne peut ni être paraphrasé ni résumé. Il est en quelque sorte non extractible de l'expérience, même si bien sûr l'expérience elle-même interagit avec toutes nos autres expériences. Par ce supplément de savoir-faire ou de sensibilité psychologique, relationnel, émotif et moral qu'elles nous livrent, les expériences d'immersion dans des récits de fiction sont capables de nous détourner de boucles réactionnelles courtes intempestives, de nous amener à suspendre notre jugement, à peser des évaluations axiologiques, à simuler des réactions affectives ou des engagements pulsionnels sans que ces expérimentations ne soient directement sanctionnées par le réel. Elles ont donc des effets dans le réel, mais on ne peut pas dire que ces effets soient induits par leur caractère de vérité déclarative référentielle ou contrefactuelle, singulière ou générale, mais plutôt par le fait qu'ils sont vécus mentalement sur le mode d'une expérience vécue.

Je ne sais pas dans quelle mesure ce qui vaut pour le récit de fiction vaut aussi pour d'autres types de discours littéraire. Par ailleurs, il est évident que la question d'une éventuelle valeur de vérité métaphorique (par exemple selon la perspective de Goodman) reste ouverte. Mais il me semble qu'il faut pour le moins se demander si, eu égard à la question de la vérité représentationnelle, l'étude de la littérature permet de rendre entièrement compte de la fonction cognitive des pratiques littéraires. 